\title{
Safety and feasibility of switching from phenytoin to levetiracetam monotherapy for glioma-related seizure control following craniotomy: a randomized phase II pilot study
}

\author{
Daniel A. Lim · Phiroz Tarapore · Edward Chang • \\ Marlene Burt · Lenna Chakalian · Nicholas Barbaro • \\ Susan Chang · Kathleen R. Lamborn · Michael W. McDermott
}

Received: 19 October 2008/Accepted: 30 December 2008/Published online: 24 January 2009

(C) The Author(s) 2009. This article is published with open access at Springerlink.com

\begin{abstract}
Seizures are common in patients with gliomas, and phenytoin (PHT) is frequently used to control tumorrelated seizures. PHT, however, has many undesirable side effects (SEs) and drug interactions with glioma chemotherapy. Levetiracetam (LEV) is a newer antiepileptic drug (AED) with fewer SEs and essentially no drug interactions. We performed a pilot study testing the safety and feasibility of switching patients from PHT to LEV monotherapy for postoperative control of glioma-related seizures. Over a 13-month period, 29 patients were randomized in a 2:1 ratio to initiate LEV therapy within $24 \mathrm{~h}$ of surgery or to continue PHT therapy. 6 month follow-up data were available for 15 patients taking LEV and for 8 patients taking PHT. In the LEV group, 13 patients (87\%) were seizure-free. In the PHT group, 6 patients $(75 \%)$ were seizure-free. Reported SEs at 6 months was as follows (\%LEV/\%PHT group): dizziness (0/14), difficulty with coordination (0/29), depression (7/14) lack of energy or strength (20/43), insomnia (40/43), mood instability (7/0). The pilot data presented here suggest that it is safe to switch patients from PHT to LEV monotherapy following craniotomy for supratentorial glioma. A large-scale, double-blinded, randomized control trial of LEV versus PHT is required to determine seizure control equivalence and better assess differences in SEs.
\end{abstract}

D. A. Lim - P. Tarapore - E. Chang - M. Burt · L. Chakalian ·

N. Barbaro $\cdot$ S. Chang $\cdot$ K. R. Lamborn .

M. W. McDermott $(\square)$

Department of Neurological Surgery, University of California, 505 Parnassus Ave. M779, San Francisco, CA 94143, USA

e-mail: mcdermottm@neurosurg.ucsf.edu

D. A. Lim

Surgical Service, Department of Veterans Affairs Medical Center,

San Francisco, CA, USA

e-mail: limd@neurosurg.ucsf.edu
Keywords Seizure - Anticonvulsant · Glioma · Levitiracetam · Phenytoin · Craniotomy

\section{Introduction}

At diagnosis, $\sim 20-40 \%$ of all brain tumor patients will have had a seizure [1] that may result in morbidity and decreased quality of life [2]. Thus, treatment with antiepileptic drugs (AEDs) is clearly indicated for brain tumor patients with preoperative tumor-related seizures [3-5]. Surgical treatment of brain tumors is also associated with a high risk of postoperative seizures. Approximately, onethird of patients have seizures after tumor resection [6-8], and in the case of supratentorial craniotomy, $20 \%-50 \%$ of patients have $\geq 1$ seizure $[9,10]$.

Brain tumor patients who experience tumor-related seizures are often placed on phenytoin (PHT) therapy. Although there are no formal guidelines, it is common practice to continue PHT after craniotomy in these patients. However, PHT has many side effects (SEs) and undesirable interactions with drugs used to treat brain tumors [1, 1114] which can complicate the pharmacological treatment of these tumors.

Levetiracetam (LEV) is a newer AED that possesses a different pharmacological profile from that of PHT, with no effects on liver enzymes and no known effect on the kinetics of other medications $[15,16]$. Furthermore, other medications including enzyme-inducing AEDs have essentially no effect on LEV pharmacokinetics [15, 17]. In retrospective studies, LEV has been found to be effective and well tolerated as add-on or monotherapy in patients with either primary or metastatic brain tumors [18, 19]. Prospectively, LEV has been studied mostly as add-on therapy for persistent tumor-related seizures [20, 21], and 
for this purpose, LEV appears to be safe and feasible and with few SEs.

Ideally, patients with brain tumor-related seizures would achieve seizure control with an AED that does not negatively interact with adjunctive chemotherapy and has few SEs. However, PHT is still commonly used as a first-line AED in brain tumor patients, and conversion of PHT therapy to LEV monotherapy has not been studied. Therefore, we prospectively studied the safety and feasibility of converting PHT to LEV monotherapy after craniotomy for glioma resection in patients with a history of tumor-related seizures.

\section{Materials and methods}

Key patient inclusion criteria for the study included: seizure history attributable to supratentorial glioma, PHT monotherapy for seizure prophylaxis, planned craniotomy as standard management, $\leq 1$ previous resection, $>18$ years of age, Karnofosky performance scale of $>70$, and no other co-morbidities. Exclusion criteria included: non-glioma cancer, pregnancy or breast-feeding, seizures unrelated to the suspected glioma, use of anticonvulsants or AEDs other than PHT, $>1$ generalized seizure per day, and prior interstitial brachytherapy. Preoperative labs were examined to ensure that patients had a $\mathrm{WBC}>3.4 \times 10^{9} / 1$, platelets $>100,000 / 1$, hemoglobin $>10 \mathrm{~g} / \mathrm{dl}$, INR $<1.3$, and serum creatinine $<1.5 \mathrm{mg} / \mathrm{dl}$.

We had planned to enroll 60 patients in this study (40 patients in the LEV group, 20 in the PHT group). This study was designed to be a feasibility study for a potential larger trial. We designed this trial to have met the "feasibility" requirement if seizure control was no more than $20 \%$ points worse than historical controls. Because of a diminishing accrual rate, we closed the study after the enrollment of 29 patients. These 29 patients were enrolled over a 13-month period. After signing an approved Institutional Review Board consent form, patients were first stratified into two groups: (1) those with no prior craniotomy, and (2) those with a history of one craniotomy. Within each stratification group, patients were randomized in a $2: 1$ ratio to initiate LEV therapy within $24 \mathrm{~h}$ of surgery or to continue PHT therapy, respectively (Fig. 1). Patients randomized to PHT therapy had serum levels confirmed to be in the therapeutic range (10-20 mg/dl) by the first postoperative day (POD1); PHT dosages were adjusted, if necessary. Patient randomized to LEV therapy were started on an oral regimen of LEV $1,000 \mathrm{mg}$ twice a day within $24 \mathrm{~h}$ of surgery; in these patients, PHT was tapered off in the following manner: $100 \%$ of preoperative PHT regimen on POD0, $75 \%$ of PHT regimen on $\mathrm{POD} 1,50 \%$ of $\mathrm{PHT}$ regimen on $\mathrm{POD} 2$, and PHT therapy was discontinued on POD3.
Patients were to be followed postoperatively for 6 months, during which their medical charts were reviewed and two standardized telephone surveys were conducted at $\sim$ 3-month intervals. Patients were provided with seizure diaries and a 24-h pager number to alert study personnel of any seizures or AED-related SEs. Seizure frequency data were based on patient-initiated contact of study personnel (through use of the 24-h pager), the telephone surveys, and review of medical charts. The primary end point was the proportion of patients who were seizure free 6 months after tumor resection. Data on AED-related SEs were collected with use of the telephone survey specifically addressing the SEs listed in Table 3.

\section{Results}

Characteristics of the study population are summarized in Table 1. Although there were some demographic differences between the two treatment groups, the majority of the study patients were male, suffered from generalized seizures, were diagnosed with glioblastoma, and all underwent craniotomy at the start of the study. One LEV subject died of the primary pathology before the first evaluation. Four other LEV subjects were withdrawn from the study by other physicians who preferred different AEDs; one patient was withdrawn by the neurosurgeon because the patient was a participant in another neurooncology trial, and PHT was the standard anticonvulsant in that study; the other three patients were withdrawn from this study by the neurosurgeon because of a personal preference for PHT as the anticonvulsant. One PHT subject could not be located after hospital discharge.

Fifteen patients in the LEV group and eight patients in the PHT group were assessed for the primary end point of seizure-freedom at 6 months (Table 2). Thirteen LEV patients $(87 \%)$ were free from seizure compared with six PHT patients $(75 \%)$. During the study period, all patients with follow-up were at least Engel Class 2 (rare seizures). Of the two patients who experienced rare seizures in the LEV group, one patient had stopped taking LEV, and this patient did not suffer additional seizures after resuming therapy; the other patient achieved seizure control with an increased dosage of $\operatorname{LEV}$ (1,500 mg twice a day). Most (11/15) LEV patients remained on the initial dose of $1,000 \mathrm{mg}$ twice a day. Three LEV patients were able to lower their dosage to $500 \mathrm{mg}$ twice a day without compromising their complete seizure control. PHT dosages were between 300 and $400 \mathrm{mg}$ per day given as single dose. No patient required hospitalization for seizures or seizure control.

Table 3 shows the SEs reported in the telephone questionnaires administered $\sim 3$ and 6 months after surgery. 


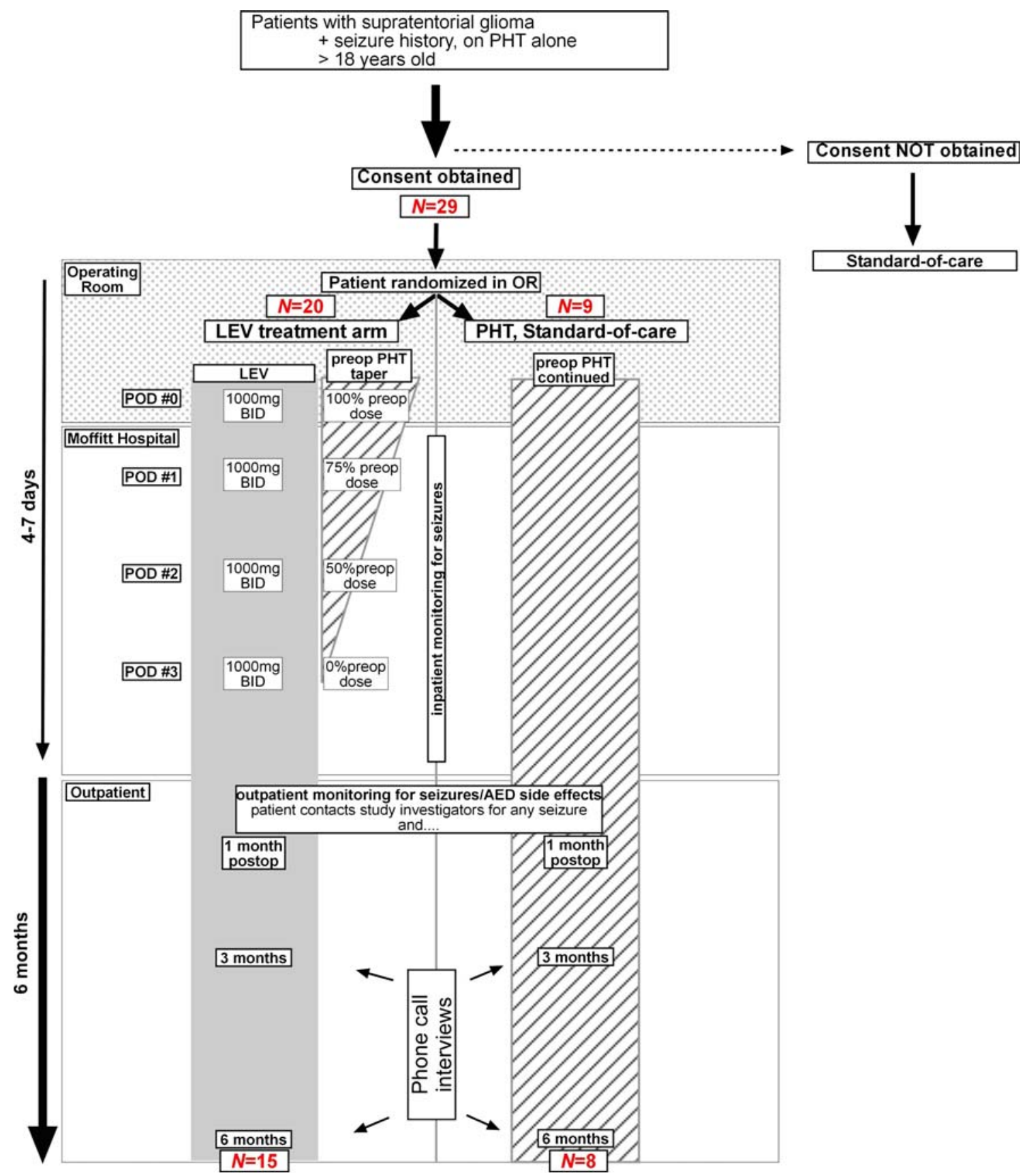

Fig. 1 Schematic showing trial design. Patients in the LEV treatment arm (left) were tapered off of PHT therapy over the course of 3 days. See materials and methods for details

The most prominent difference between the LEV and PHT groups in terms of SEs was difficulty with coordination: no patient in the LEV group reported this difficulty, compared to $4(57 \%)$ and $2(29 \%)$ of the PHT group at 3 and 6 months, respectively. The one patient taking LEV $1,500 \mathrm{mg}$ twice a day did not report more SEs than those taking $500 \mathrm{mg}$ twice a day, suggesting that LEV dosage does not correlate with increased SEs. No SEs in any study patient resulted in a hospitalization or withdrawal from study.

\section{Discussion}

An ideal AED for brain tumor patients would be one that has few SEs and does not interfere with medications commonly used in brain tumor treatment. While PHT is very commonly used for seizure control in brain tumor patients, it has many undesirable pharmacological qualities. PHT induces the metabolism of the steroid dexamethasone [11, 13], and dexamethasone decreases PHT serum levels [22]. Certain chemotherapeutic agents 
Table 1 Characteristics of study population
DNET dysembryoplastic neuroepithelial tumor; $P X A$ pleomorphic xanthoastrocytoma; $\mathrm{WHO}$ World Health Organization (brain tumor grading scale)

\begin{tabular}{|c|c|c|c|}
\hline & $\begin{array}{l}\text { Total patients } \\
\mathrm{n}(\%)\end{array}$ & $\begin{array}{l}\text { LEV patients } \\
\mathrm{n}(\% \text { of arm })\end{array}$ & $\begin{array}{l}\text { PHT patients } \\
\mathrm{n}(\% \text { of arm })\end{array}$ \\
\hline Subjects enrolled & $29(100)$ & $20(69)$ & $9(31)$ \\
\hline Subjects with primary end-point follow-up & $23(79)$ & $15(75)$ & $8(89)$ \\
\hline \multicolumn{4}{|l|}{ Demographics } \\
\hline Female & $6(26)$ & $6(40)$ & 0 \\
\hline Median age (range) & $45(20-83)$ & $46(20-56)$ & $39(32-83)$ \\
\hline \multicolumn{4}{|l|}{ Seizure type at presentation } \\
\hline Simple partial & $7(30)$ & $6(40)$ & $1(13)$ \\
\hline Complex partial & $5(22)$ & $1(7)$ & $4(50)$ \\
\hline Generalized & $11(48)$ & $8(53)$ & $3(38)$ \\
\hline \multicolumn{4}{|l|}{ Surgery } \\
\hline Craniotomy before study surgery & $7(30)$ & $4(27)$ & $3(38)$ \\
\hline \multicolumn{4}{|l|}{ Craniotomy for study } \\
\hline Gross total resection & $13(56)$ & $10(67)$ & $3(38)$ \\
\hline Subtotal resection & $10(43)$ & $5(33)$ & $5(63)$ \\
\hline \multicolumn{4}{|l|}{ Final tumor pathology } \\
\hline \multicolumn{4}{|l|}{ WHO grade 1} \\
\hline DNET & $1(4)$ & $1(7)$ & 0 \\
\hline \multicolumn{4}{|l|}{ WHO grade 2} \\
\hline Astrocytoma & $3(13)$ & $2(13)$ & $1(13)$ \\
\hline Oligodendroglioma & $2(9)$ & $1(7)$ & $1(13)$ \\
\hline PXA & $1(4)$ & $1(7)$ & 0 \\
\hline \multicolumn{4}{|l|}{ WHO grade 3} \\
\hline Astrocytoma & $4(17)$ & $4(27)$ & 0 \\
\hline Oligodendroglioma & $1(4)$ & 0 & $1(13)$ \\
\hline Oligoastrocytoma & $2(9)$ & $1(7)$ & $1(13)$ \\
\hline \multicolumn{4}{|l|}{ WHO grade 4} \\
\hline Glioblastoma & 9 (39) & $6(40)$ & $3(38)$ \\
\hline
\end{tabular}

Table 2 Postoperative seizure status at 6 months

\begin{tabular}{lcl}
\hline Seizure status (Engel score) & $\begin{array}{l}\text { LEV patients } \\
\mathrm{n}(\%)\end{array}$ & $\begin{array}{l}\text { PHT patients } \\
\mathrm{n}(\%)\end{array}$ \\
\hline $\mathrm{n}$ at follow-up & 15 & 8 \\
Class 1: seizure free & $13(87)$ & $6(75)$ \\
Class 2: rare seizures & $2(12)$ & $2(25)$ \\
Class 3: meaningful improvement & 0 & 0 \\
Class 4: no improvement & 0 & 0 \\
\hline
\end{tabular}

also lower PHT levels [12], and, vice versa, PHT can accelerate the metabolism of a variety of chemotherapy drugs including nitrosoureas, paclitaxel, 9-aminocamptothecin, thiotepa, topotecan, and irinotecan ([1, 14] and references therein). In terms of SEs, PHT adversely affects cognition [23, 24], which is often already depressed in brain tumor patients. Furthermore, brain tumor patients taking PHT are at increased risk of drug rashes [25], including Stevens-Johnson syndrome [26], an immunemediated rash associated with severe morbidity and death.
Because LEV does not have any significant drug interactions, this AED poses fewer complications for the pharmacotherapy of brain tumors. A retrospective study of 34 patients with primary brain tumors suggested that LEV is effective and well tolerated for tumor-related seizure control, especially as add-on therapy to other AEDs [19]. Prospectively, LEV has been studied as add-on therapy for seizure control in brain tumor patients. In a cohort of 25 patients with primary brain tumors, Wagner et al. [21] found that add-on LEV reduced seizure frequency by more than $50 \%$ in 13 of the patients who experienced persistent seizures. Maschio et al. [20] prospectively followed 19 patients with primary brain tumors who had LEV added to existing AED therapy on account of persisting seizures; in that study, 14 patients had improvement in seizure control. There have been no reports of serious SEs of LEV therapy in any of the studies of brain tumor patients to date. For the reasons described above, safe conversion of PHT to LEV monotherapy may provide significant clinical advantages for the post-surgical medical treatment of primary brain tumors. 
Table 3 Results of patient questionnaire on SEs

\begin{tabular}{|c|c|c|c|c|}
\hline & \multicolumn{2}{|c|}{3 months postoperation } & \multicolumn{2}{|c|}{6 months postoperation } \\
\hline & $\begin{array}{l}\text { LEV patients } \\
\mathrm{n}(\%)\end{array}$ & $\begin{array}{l}\text { PHT patients } \\
\mathrm{n}(\%)\end{array}$ & $\begin{array}{l}\text { LEV patients } \\
\mathrm{n}(\%)\end{array}$ & $\begin{array}{l}\text { PHT patients } \\
\mathrm{n}(\%)\end{array}$ \\
\hline Number of respondents & 15 & 7 & 15 & 7 \\
\hline \multicolumn{5}{|l|}{ Questions regarding potential SES } \\
\hline \multicolumn{5}{|c|}{ Have you been experiencing any of the following symptoms? } \\
\hline Dizziness & $1(7)$ & 0 & 0 & $1(14)$ \\
\hline Headache & $1(7)$ & $1(14)$ & $1(7)$ & $1(14)$ \\
\hline Difficulty with coordination & 0 & $4(57)$ & 0 & $2(29)$ \\
\hline Excessive sleepiness & $3(20)$ & $2(29)$ & $3(20)$ & $2(29)$ \\
\hline Enlarged gums & 0 & 0 & 0 & 0 \\
\hline Depression & $1(7)$ & $1(14)$ & $1(7)$ & $1(14)$ \\
\hline Difficulty sleeping & $5(33)$ & $1(14)$ & $6(40)$ & $3(43)$ \\
\hline Itchiness & 0 & $1(14)$ & 0 & 0 \\
\hline Nausea & 0 & 0 & 0 & 0 \\
\hline Vomiting & 0 & 0 & 0 & 0 \\
\hline Tingling, "pins and needles" sensation & 0 & $1(14)$ & 0 & 0 \\
\hline Nervousness & $1(7)$ & $1(14)$ & 0 & 0 \\
\hline Emotional instability & $2(13)$ & 0 & $1(7)$ & 0 \\
\hline Slurred speech & $1(7)$ & 0 & $2(13)$ & 0 \\
\hline Lack of Energy or Strength & $4(27)$ & $4(57)$ & $3(20)$ & $3(43)$ \\
\hline Constipation & 0 & $1(14)$ & 0 & 0 \\
\hline Difficulty keeping balance & 0 & 0 & 0 & 0 \\
\hline Rash & 0 & 0 & 0 & 0 \\
\hline $\begin{array}{l}\text { Have you been experiencing any other SEs } \\
\text { that you attribute to your medication? }\end{array}$ & $3(20)$ & $2(29)$ & $2(13)$ & $2(29)$ \\
\hline
\end{tabular}

In our study population, LEV and PHT groups had similar proportions of seizure-free patients $(87 \%$ and $75 \%$, respectively) at 6 months of follow-up. These seizure control rates are in line with those observed in a retrospective analysis of low grade glioma patients treated at this institution; of 253 patients who had tumor-related seizures, 169 (67\%) were seizure-free 6 months after craniotomy for tumor resection [8]. Thus, LEV and PHT groups had expected levels of seizure control. Furthermore, no patient in our study had seizures refractory to either LEV or PHT therapy.

LEV and PHT were both well tolerated in our study population. Although difficulty with coordination is a potential SE for both LEV and PHT, no patient in the LEV study group (0/15) admitted to experiencing this difficulty while more than half of the PHT group (4/7) at 3 months reported this SE. Other known LEV SEs are somnolence and behavioral abnormalities. Both PHT and LEV groups had similar proportions of patients reporting somnolence. One subject in the LEV group reported feeling unusually hostile towards others after starting LEV therapy; however, this behavioral change did not require the discontinuation of LEV. No PHT study subject admitted to experiencing any emotional instability at any time point. In placebo-controlled trials of LEV for partialonset epilepsy, LEV was associated with a $0.7 \%$ incidence of psychosis as compared to $0.2 \%$ for placebo patients. Although Maschio et al. reported that there were no LEVrelated SEs observed in their study of primary brain tumor patients, Wagner et al. did report one patient in their study of 26 who developed a psychosis 4 weeks after initiating LEV therapy which resulted in discontinuation of this AED. Given the report of psychosis in Wagner et al. and our findings of emotional instability in up to $13 \%$ of our LEV group as compared to none in the PHT group (Table 2), we suggest that it would be important to evaluate brain tumor patients on LEV therapy for potential behavioral problems.

Our pilot data presented here suggest that it is safe and feasible to switch from PHT to LEV monotherapy after craniotomy for glioma-related seizures. Due to the small size of our study, the data presented here cannot serve as an official endorsement of LEV efficacy in this patient population. A large-scale, double-blinded, randomized controlled trial is warranted to compare PHT and LEV for seizure control equivalence and SE profiles. 
Acknowledgment This work was supported by a Young Investigator Research Program award to D.A.L. and M.W.M. from UCB Pharma.

Open Access This article is distributed under the terms of the Creative Commons Attribution Noncommercial License which permits any noncommercial use, distribution, and reproduction in any medium, provided the original author(s) and source are credited.

\section{References}

1. Glantz MJ, Cole BF, Forsyth PA, Recht LD, Wen PY, Chamberlain MC, Grossman SA, Cairncross JG (2000) Practice parameter: anticonvulsant prophylaxis in patients with newly diagnosed brain tumors. Report of the Quality Standards Subcommittee of the American Academy Of Neurology. Neurology 54:1886-1893

2. Klein M, Engelberts NH, van der Ploeg HM, Kasteleijn-Nolst Trenite DG, Aaronson NK, Taphoorn MJ, Baaijen H, Vandertop WP, Muller M, Postma TJ, Heimans JJ (2003) Epilepsy in lowgrade gliomas: the impact on cognitive function and quality of life. Ann Neurol 54:514-520. doi:10.1002/ana.10712

3. Holland KD (2001) Efficacy, pharmacology, and adverse effects of antiepileptic drugs. Neurol Clin 19:313-345. doi:10.1016/ S0733-8619(05)70021-9

4. Schachater SC (2001) Epilepsy. Neurol Clin 19:57-78. doi: 10.1016/S0733-8619(05)70005-0

5. Wen PY, Marks PW (2002) Medical management of patients with brain tumors. Curr Opin Oncol 14:299-307. doi:10.1097/ 00001622-200205000-00008

6. Chang EF, Keles GE, Chang SM, Lamborn KL, Barbaro NM, Berger MS (2005) Seizure characteristics and post-operative seizure control in patients with low-grade gliomas. Congress of Neurological Surgeons (abstract) 2005. Los Angeles, CA, USA

7. Franceschetti S, Binelli S, Casazza M, Lodrini S, Panzica F, Pluchino F, Solero CL, Avanzini G (1990) Influence of surgery and antiepileptic drugs on seizures symptomatic of cerebral tumours. Acta Neurochir (Wien) 103:47-51. doi:10.1007/BF01 420191

8. Chang EF, Potts MB, Keles GE, Lamborn KR, Chang SM, Barbaro NM, Berger MS (2008) Seizure characteristics and control following resection in 332 patients with low-grade gliomas. J Neurosurg 108:227-235. doi:10.3171/JNS/2008/108/ $2 / 0227$

9. North JB, Penhall RK, Hanieh A, Frewin DB, Taylor WB (1983) Phenytoin and postoperative epilepsy. A double-blind study. J Neurosurg 58:672-677

10. Shaw MD, Foy PM (1991) Epilepsy after craniotomy and the place of prophylactic anticonvulsant drugs: discussion paper. J R Soc Med 84:221-223

11. Chalk JB, Ridgeway K, Brophy T, Yelland JD, Eadie MJ (1984) Phenytoin impairs the bioavailability of dexamethasone in neurological and neurosurgical patients. J Neurol Neurosurg Psychiatry 47:1087-1090. doi:10.1136/jnnp.47.10.1087

12. Grossman SA, Sheidler VR, Gilbert MR (1989) Decreased phenytoin levels in patients receiving chemotherapy. Am J Med 87:505-510

13. Werk EE Jr, Choi Y, Sholiton L, Olinger C, Haque N (1969) Interference in the effect of dexamethasone by diphenylhydantoin. N Engl J Med 281:32-34

14. van Breemen MS, Wilms EB, Vecht CJ (2007) Epilepsy in patients with brain tumours: epidemiology, mechanisms, and management. Lancet Neurol 6:421-430. doi:10.1016/S14744422(07)70103-5

15. Patsalos PN (2004) Clinical pharmacokinetics of levetiracetam. Clin Pharmacokinet 43:707-724. doi:10.2165/00003088-200443 110-00002

16. Welty TE, Gidal BE, Ficker DM, Privitera MD (2002) Levetiracetam: a different approach to the pharmacotherapy of epilepsy. Ann Pharmacother 36:296-304. doi:10.1345/aph.1A032

17. Contin M, Albani F, Riva R, Baruzzi A (2004) Levetiracetam therapeutic monitoring in patients with epilepsy: effect of concomitant antiepileptic drugs. Ther Drug Monit 26:375-379. doi: 10.1097/00007691-200408000-00006

18. Newton HB, Dalton J, Goldlust S, Pearl D (2007) Retrospective analysis of the efficacy and tolerability of levetiracetam in patients with metastatic brain tumors. J Neurooncol 84:293-296. doi:10.1007/s11060-007-9373-8

19. Newton HB, Goldlust SA, Pearl D (2006) Retrospective analysis of the efficacy and tolerability of levetiracetam in brain tumor patients. J Neurooncol 78:99-102. doi:10.1007/s11060-0059070-4

20. Maschio M, Albani F, Baruzzi A, Zarabla A, Dinapoli L, Pace A, Pompili A, Carapella CM, Occhipinti E, Jandolo B (2006) Levetiracetam therapy in patients with brain tumour and epilepsy. J Neurooncol 78:157-160

21. Wagner GL, Wilms EB, Van Donselaar CA, Vecht ChJ (2003) Levetiracetam: preliminary experience in patients with primary brain tumours. Seizure 12:585-586. doi:10.1016/S1059-1311 (03)00096-7

22. Lawson LA, Blouin RA, Smith RB, Rapp RP, Young AB (1981) Phenytoin-dexamethasone interaction: a previously unreported observation. Surg Neurol 16:23-24. doi:10.1016/S0090-3019 (81)80054-7

23. Akaho R (1996) The effects of antiepileptic drugs on cognition in normal volunteers. Psychiatry Clin Neurosci 50:61-69. doi: 10.1111/j.1440-1819.1996.tb01665.x

24. Drane DL, Meador KJ (2002) Cognitive and behavioral effects of antiepileptic drugs. Epilepsy Behav 3:49-53. doi:10.1016/S15255069(02)00502-9

25. Mamon HJ, Wen PY, Burns AC, Loeffler JS (1999) Allergic skin reactions to anticonvulsant medications in patients receiving cranial radiation therapy. Epilepsia 40:341-344. doi:10.1111/ j.1528-1157.1999.tb00715.x

26. Delattre JY, Safai B, Posner JB (1988) Erythema multiforme and Stevens-Johnson syndrome in patients receiving cranial irradiation and phenytoin. Neurology 38:194-198 